\title{
Simultaneous targeting of HER family pro- survival signaling with Pan-HER antibody mixture is highly effective in TNBC: a preclinical trial with PDXs
}

Tejaswini P. Reddy ${ }^{1,2+}$, Dong S. Choi ${ }^{1 \dagger}$, Ann C. Anselme ${ }^{1,2}$, Wei Qian ${ }^{1}$, Wen Chen ${ }^{1}$, Johan Lantto ${ }^{3}$, Ivan D. Horak ${ }^{3}$, Michael Kragh ${ }^{2}$, Jenny C. Chang ${ }^{1}$ and Roberto R. Rosato ${ }^{1 *}$

\begin{abstract}
Background: The human epidermal growth factor receptor (HER) family, notably EGFR, is overexpressed in most triple-negative breast cancer (TNBC) cases and provides cancer cells with compensatory signals that greatly contribute to the survival and development of resistance in response to therapy. This study investigated the effects of Pan-HER (Symphogen, Ballerup, Denmark), a novel mixture of six monoclonal antibodies directed against members of the HER family EGFR, HER2, and HER3, in a preclinical trial of TNBC patient-derived xenografts (PDXs).

Methods: Fifteen low passage TNBC PDX tumor samples were transferred into the right mammary fat pad of mice for engraftment. When tumors reached an average size of $100-200 \mathrm{~mm}^{3}$, mice were randomized ( $n \geq 6$ per group) and treated following three 1-week cycles consisting of three times/week intraperitoneal (IP) injection of either formulation buffer (vehicle control) or Pan-HER $(50 \mathrm{mg} / \mathrm{kg}$ ). At the end of treatment, tumors were collected for Western blot, RNA, and immunohistochemistry analyses.

Results: All 15 TNBC PDXs were responsive to Pan-HER treatment, showing significant reductions in tumor growth consistent with Pan-HER-mediated tumor downmodulation of EGFR and HER3 protein levels and significantly decreased activation of associated HER family signaling pathways AKT and ERK. Tumor regression was observed in five of the models, which corresponded to those PDX tumor models with the highest level of HER family activation. Conclusions: The marked effect of Pan-HER in numerous HER family-dependent TNBC PDX models justifies further studies of Pan-HER in TNBC clinical trials as a potential therapeutic option.
\end{abstract}

Keywords: HER family, EGFR, Triple-negative breast cancer, HER2, HER3, PDX, Pan-HER

\footnotetext{
* Correspondence: rrrosato@houstonmethodist.org

${ }^{\dagger}$ Tejaswini P. Reddy and Dong S. Choi contributed equally to this work.

'Houston Methodist Cancer Center, Houston Methodist Hospital, Houston,

TX 77030, USA

Full list of author information is available at the end of the article
}

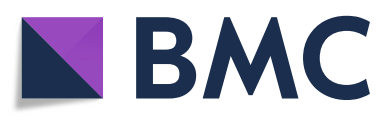

(- The Author(s). 2020 Open Access This article is licensed under a Creative Commons Attribution 4.0 International License, which permits use, sharing, adaptation, distribution and reproduction in any medium or format, as long as you give appropriate credit to the original author(s) and the source, provide a link to the Creative Commons licence, and indicate if changes were made. The images or other third party material in this article are included in the article's Creative Commons licence, unless indicated otherwise in a credit line to the material. If material is not included in the article's Creative Commons licence and your intended use is not permitted by statutory regulation or exceeds the permitted use, you will need to obtain permission directly from the copyright holder. To view a copy of this licence, visit http://creativecommons.org/licenses/by/4.0/. The Creative Commons Public Domain Dedication waiver (http://creativecommons.org/publicdomain/zero/1.0/) applies to the data made available in this article, unless otherwise stated in a credit line to the data. 


\section{Background}

As a heterogeneous disease, breast cancer is clinically classified by taking into account the expression of estrogen receptor alpha $(\mathrm{ER} \alpha)$, progesterone receptor $(\mathrm{PR})$, and the presence/amplification status of the oncogenic human epidermal growth factor receptor 2 (HER2) [1]. Triple-negative breast cancer (TNBC), i.e., those not expressing ER, PR, or HER2, represent 10-20\% of all cases, have poorer prognosis than HER2 ${ }^{+}$or hormone receptor-positive tumors, and are generally characterized by an aggressive clinical course [2]. Furthermore, due to the lack of druggable targets including HER2 and ER, the main therapeutic options remain surgery and systemic chemotherapies, either individually or combined [reviewed in [3]]. Importantly, epidermal growth factor receptor (EGFR) is more often overexpressed in TNBC than in other breast cancer subtypes, making this a possible target for therapeutic intervention.

The present study investigated the effects of Pan-HER, a novel mixture of six monoclonal antibodies (mAbs) directed against members of the human epidermal growth factor receptor (HER) family EGFR/HER1, HER2, and HER3 [4], in a preclinical trial of TNBC patient-derived xenografts (PDXs). The HER family, notably EGFR, is well recognized for its pro-oncogenic activity that upon activation by corresponding ligands lead to receptor dimerization [5]. These effects are mediated through downstream signals including PI3K/AKT, RAS/RAF/MEK/ ERK1/2, JAK/STAT3, and PLC $\gamma$-pathways ([5]. Several cancer types have been shown to be associated with either mutation or increased expression of the HER family members, including breast, lung, stomach, colorectal, head and neck, pancreatic carcinomas, and glioblastoma \{Roskoski, 2014 \#2300, [6]). Furthermore, accumulating evidence shows that the HER family is important in providing cancer cells with compensatory signals that greatly contribute to the development of resistance and survival in response to therapy [7-9]. Pan-HER was designed based on the hypothesis that simultaneous inhibition and/or downmodulation of multiple members of the HER family may result in effective disruption of tumor growth, preventing the HERdriven cell proliferation, survival, angiogenesis, and invasion [10]. Earlier studies, including clinical trials [11, 12], explore the concept of targeting simultaneously either HER family members or combining antibody-based therapy with kinase inhibitors $[13,14]$ and have set the stage for further investigation of this mechanistic-related concept. In this context, the potential therapeutic advantage of Pan-HER may reside in its ability to simultaneously target all three receptor tyrosine kinases (RTKs), which may in turn block, or at least significantly delay, the appearance of survival and escape mechanisms $[4,15]$.

The effect of Pan-HER has been investigated in a number of cell lines and xenografts representing a diverse number of cancer types including head and neck, lung [16, 17],
HER $^{+}$breast [18], and other malignancies shown to have a dependency on one or more of the targets, i.e., EGFR, HER2, or HER3 [4]. Pan-HER demonstrated stronger activity than single mAbs directed against single HER family members and was capable of overcoming acquired resistance due to increased ligand expression [4]. Here, we present the results of a preclinical trial of Pan-HER performed in 15 TNBC PDX models. Our results show significant antitumor activity by Pan-HER in all PDX tumor models evaluated, with a noticeable therapeutic response in those TNBC tumors whose dependency on the HER family appeared to be most pronounced.

\section{Methods \\ Reagents}

Pan-HER, a mixture of six monoclonal antibodies directed against each of the HER family members EGFR, HER2, and HER3, was generously provided by Symphogen A/S (Denmark) $[4,15,19]$. Pan-HER is formed by the combination of three sets of two antibodies each targeting nonoverlapping epitopes of EGFR, HER2, and HER3.

\section{Mice}

All protocols involving mice followed standard regulations and were approved by the Houston Methodist Research Institute Institutional Animal Care and Use Committee (IACUC). Female immunodeficient SCID/beige mice (Envigo, Indianapolis, IN) were used as the recipient strain to engraft PDXs. PDXs were originally derived by transplantation of a fresh treatment-naïve patient breast tumor biopsy into the cleared mammary gland fat pad of immunocompromised mice [20]. Low passage TNBC PDX tumor samples $(2 \times 2 \times 2 \mathrm{~mm}$; Additional file 1: Table S1) were transferred into the right mammary fat pad of mice for engraftment. Mouse body weights were recorded as an indication of the animals' health status; tumor volumes were measured and calculated twice weekly following the formula $\left[0.5 \times(\right.$ long dimension $\left.) \times(\text { short dimension })^{2}\right]$. When tumors reached an average size of 100 to 200 $\mathrm{mm}^{3}$, mice were randomized ( $n \geq 6$ per group) and used to determine the response to treatment. Tumor volume fold change was calculated based on the baseline tumor volumes for each arm.

\section{Gene expression and data analyses}

Relative expression levels or genetic alterations/modifications corresponding to HER family members (EGFR and HER3) or associated signaling pathway (i.e., focal adhesion kinase [FAK] and phosphatase and tensin homolog [PTEN]) in human breast cancer including triple-negative breast cancer, were investigated by Oncomine Cancer Microarray database analysis [21] of The Cancer Genome Atlas (TCGA); cBioPortal (Memorial Sloan Kettering Cancer Center's Computational Biology Center, New York, 
NY) and NCI-GDC Data Portal database were used to interrogate for the incidence of key genes/pathways related to the present study ( $n=8824$ patients, 9052 samples in 12 studies). Microarrays were performed using Affymetrix GeneChip U133plus 2.0. Normalization and evaluation of the data were performed as previously described [22]. The array data were evaluated using the commercial software suite, Partek Genomics Suite. Specifically, data were normalized by using the RMA (robust multichip averaging) method. Gene expression levels were analyzed on a logarithmic scale. ANOVA was used to identify differentially expressed genes. Genes with a $P$ value of less than 0.05 in each comparison were selected for further functional and pathway analyses by Ingenuity Pathway Analysis (IPA; Qiagen, Germantown, MD) tools. Patient survival analysis was obtained by using Kaplan-Meier analysis tools as previously described $[23,24]$. EGFR and NF- $\mathrm{kB}$ signaling pathway-focused real-time RT-PCR analyses were performed by using Pathway PCR Arrays (RealTimePrimers, Elkins Park, PA) and the SensiFAST SYBR No-ROX OneStep Kit (Bioline USA, Taunton, MA) according to the manufacturers' protocol. Gene expression was compared according to the $C_{\mathrm{T}}$ value. Gene expression analysis included also that performed by Ingenuity Pathway Analysis (IPA, Qiagen). The corresponding full lists of genes included in each array are described in Additional file 1: Table S2. Each array contained 88 targeted plus 8 housekeeping gene primer sets.

\section{Immunohistochemistry}

Immunohistochemistry assays were performed following well-established protocols as previously described [25]. After antigen retrieval (Tris- $\mathrm{Cl}, \mathrm{pH}$ 9.0), paraffinembedded sections of PDX tumors were incubated for 1 $\mathrm{h}$ at room temperature with the following antibodies: anti-human EGFR, clone D38B1 (Cell Signaling Technology, Danvers, MA); and anti-human HER3/ErbB3 (clone D22C5; Cell Signaling Technology).

\section{Western blot analysis}

Analysis of proteins was performed by Western blot as previously described [25]. Briefly, $30 \mu \mathrm{g}$ protein of whole cell lysates were subjected to SDS-PAGE electrophoresis in 4 to $20 \%$ polyacrylamide gels (Bio-Rad, Hercules, CA, USA). Proteins were transferred onto nitrocellulose membranes (Bio-Rad), incubated overnight at $4{ }^{\circ} \mathrm{C}$ with primary antibodies (1:1000), followed by washes and incubation with the appropriate secondary antibodies for $1 \mathrm{~h}$ (1:2000). Protein bands were developed in autoradiography films (Denville Scientific Inc., South Plainfield, $\mathrm{NJ})$. Antibodies used in this study were purchased from Cell Signaling Technology and included anti-EGF Receptor (D38B1), anti-HER3/ErbB3 (D22C5), anti-phosphoEGFR (Tyr1068; D7A5), anti-phospho-HER3/ErbB3
(Tyr1289; D1B5), anti-phospho-FAK (Tyr925), antiphospho-Stat3 (Tyr705), anti-Stat3 (79D7), antiphospho-AKT (S4737), and anti-AKT (C67E7).

\section{Statistical analysis}

All data were analyzed using GraphPad Prism (GraphPad Software, La Jolla, CA). Data are presented as mean \pm standard error of the mean. Statistical significance between two groups was analyzed by two-tailed Student's $t$ test. Experiments with more than three groups were analyzed with one-way analysis of variance (ANOVA) and Bonferroni's post hoc test. Statistical analysis of tumor volume was assessed by two-way ANOVA and Bonferroni's post hoc test. A $P$ value of less than 0.05 was considered significant.

\section{Results}

Prevalence of HER family mutations in breast cancer Using public databases and analysis tools (see "Methods") $[21,23,24]$, the prevalence of mutations and/or alterations in HER family members were investigated in a pool representing 8824 breast cancer patients (a total of 9052 samples from 12 studies), including those corresponding to TNBC. Genetic alterations were found corresponding to EGFR (3\%) and HER3 (2.4\%) (Fig. 1a). Gene expression analysis corresponding to 13 of the PDXs used in the present study at baseline (i.e., low passage, non-treated PDX tumor tissue) was performed. Three of the PDXs including BCM-3936, BCM-4913, and MC1 displayed a very similar gene expression pattern that substantially differed from the rest of samples (Additional file 2: Figure S1a). As determined by Ingenuity Pathway Analysis (IPA) comparing these three PDXs against the rest of the models, changes in genes related to the PTEN pathway, a very well-described tumor suppressor gene [26, 27], and PTK2 (FAK) were among the top molecules (Additional file 2: Figure S1b). Both genes represent key components of the AKT/PKB survival pathway, a critical downstream signaling on HER family activation [28-31]. Based in these observations, we included into the analysis of data bases both PTK2 and PTEN, both showing also marked alterations in breast cancer samples $(12 \%$ and $7 \%$, respectively; Fig. 1a). Importantly, these mutations/alterations were directly correlated with reduced overall survival (OS; Fig. 1b). Together, these data indicate a clear association between alterations in HER family members and their corresponding signaling pathways with poorer prognosis and survival in breast cancer.

\section{Evaluation of Pan-HER activity against TNBC PDXs}

In order to evaluate the activity of Pan-HER as a therapy for TNBC, a total of 15 different PDX tumor models were selected from our collection [20] to perform a preclinical trial. All of the PDXs used in this study were 
a Amplification/overexpression of EGFR, HER3, FAK (PTK2), and PTEN in TCGA Breast Cancer (8824patients)

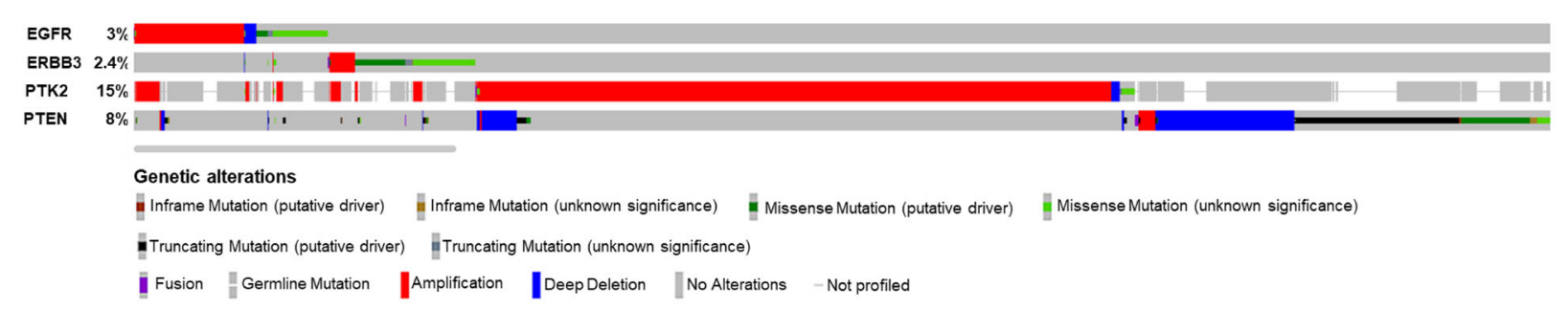

b Overall Survival Kaplan-Meier Estimate

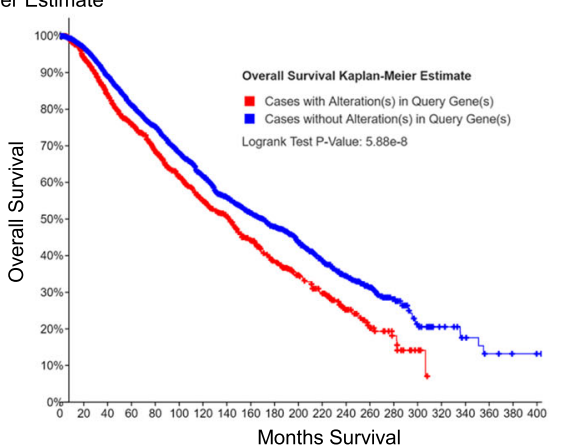

Fig. 1 Amplification/overexpression of the HER family correlates with poor prognosis in breast cancer. a Data acquired from CBioPortal (NCI-GDC Data Portal) for Cancer Genomics showing the percentages of genetic modifications of different components of the HER family among breast cancer patients (8824 breast cancer patients, a total of 9052 samples from 12 studies). b Alterations of HER family members portends significantly poorer overall survival in TNBC patients [Kaplan-Meir analysis tools: Refs. [23, 24]]

originally derived by transplantation of a fresh treatment-naïve patient breast tumor biopsy into the cleared mammary gland fat pad of immunocompromised mice [20]; they are triple negative (i.e., $\mathrm{ER}^{-}, \mathrm{PR}^{-}$, and $\mathrm{HER}^{-}$) with positive expression of both EGFR and HER3, with the exception of BCM-4664, which displays

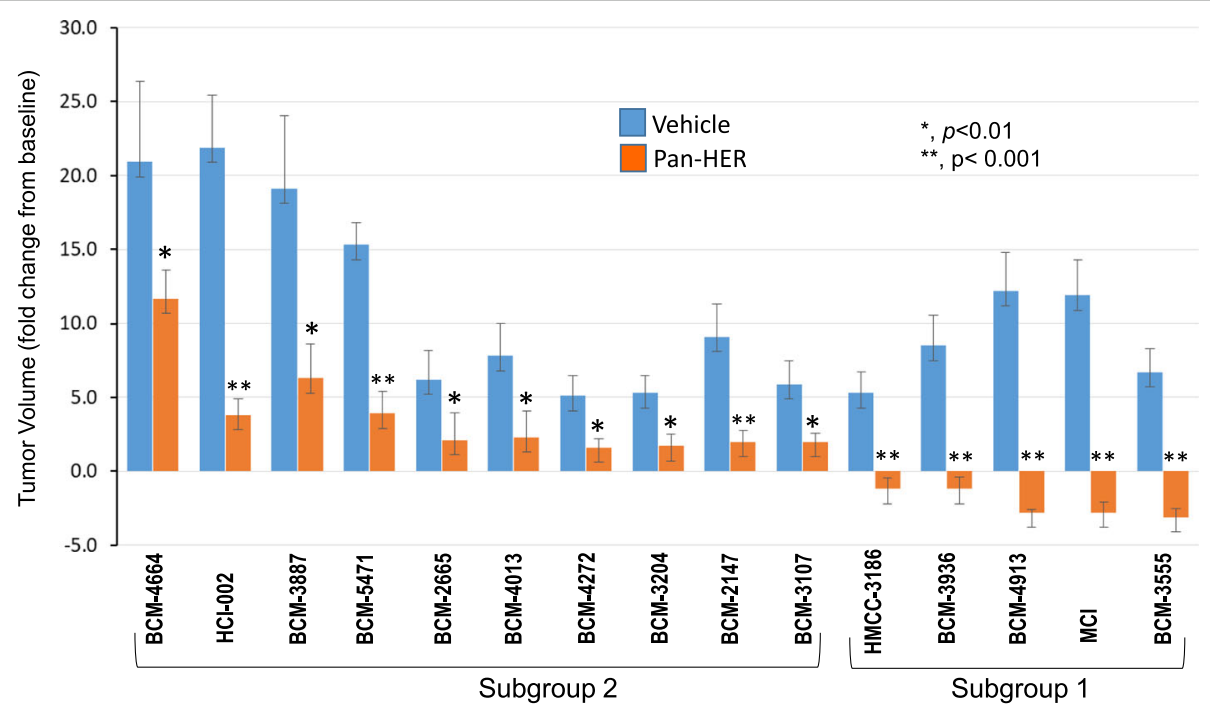

Fig. 2 Overall responses of 15 TNBC PDX models to Pan-HER therapy. Low passage TNBC PDX tumor samples were transferred into the right mammary fat pad of mice for engraftment. Once tumors reached an average size of 100-200 $\mathrm{mm}^{3}$, mice were randomized ( $n \geq 6$ per group) and used to determine the response to the treatment. Regimen treatment design followed three, 1-week cycles consisting of IP injection three times/ week of either formulation buffer (vehicle control) or Pan-HER $(50 \mathrm{mg} / \mathrm{kg}$ ). Mouse weight was recorded and tumor volumes measured and calculated, as described in "Methods", twice weekly. Tumor volume fold change was calculated based on the baseline tumor volumes for each arm. Two-way ANOVA was used for statistical analysis 
low EGFR expression and HER3- (Additional file Tables: Table S1); additional information includes, whenever available, TP53 mutations, PAM50, and Pietenpol subtype classification (Additional file Tables: Table S1) [20, 32-34]. The characterization of PDXs also included sequence analysis of tumor PDXs for mutations in PI3KCA (exons 9 and 20) and EGFR (exons 18, 19, 20, and 21) $[35,36]$. No changes and/or functionally significant alterations in the mentioned exons were found (Additional file Tables: Tables S2-4; Additional file Methods).

The treatment regimen followed three 1-week cycles consisting of an intraperitoneal (IP) injection three times per week of either formulation buffer (vehicle control) or Pan-HER $(50 \mathrm{mg} / \mathrm{kg})$, commencing once tumors had reached an average size of $100-200 \mathrm{~mm}^{3}$. The antitumor response to the Pan-HER therapy is summarized in Fig. 2. Strikingly, all 15 PDX tumor models, which had different growth rates as reflected by the fold change in tumor size within 3 weeks of starting the treatment, showed a significant reduction in tumor volume at the end of the third cycle of therapy, notably five of them which displayed tumor regression (Fig. 2). Based on these results, PDXs were divided into subgroup 1, which included those TNBC PDXs that were highly responsive (tumor regression) to treatment with Pan-HER, and the remainder, subgroup 2, that still displayed a positive response, although more moderate in terms of tumor growth inhibition (Fig. 2). Details on each of the PDX response to Pan-HER treatment are given in Additional file 2: Figures S2-S16.

The time-course response of three representative PDXs selected based on their corresponding response analysis (Fig. 2), namely BCM-4664 (fast growing, very aggressive tumor), BCM-2147 (moderate growth), and BCM-3555 (slow growth), is shown in Fig. 3. Consistent with the results shown in Fig. 2, all PDXs treated with Pan-HER displayed significant delays in tumor growth by the end of the third cycle (blue arrow; Fig. 3 and Additional file 2: Figures S2S16). PDXs BCM-4913, MC1, and BCM-3555 (subgroup 1) were followed for an extended period (128, 58, and 145 days, respectively) to determine potential tumor recurrence. In all three cases, which were among those with the best response to the treatment, no signs of recurrence were observed (Fig. 3 and Additional file 2: Figures S14-16). Overall, the preclinical trial performed in 15 different TNBC PDXs showed that treatment with Pan-HER was highly effective at controlling tumor growth, leading in some cases to complete tumor regression and no recurrence.

\section{Pan-HER activity correlates with activation of the EGFR, HER3, and associated signaling pathways}

As mentioned earlier, the differential gene expression analysis of PDXs used in this study at baseline showed that BCM-3936, BCM-4913, and MC1 (highly responsive to Pan-HER, subgroup 1), differed markedly from the rest of the PDX tumor models (Additional file 2: Figure S1a). Furthermore, IPA analysis showed that genes corresponding to both the PTEN and FAK signaling were in the top canonical pathways. Importantly, a marked reduction of genes related to the PTEN pathway, a very well-described tumor suppressor gene [26, 27], and increased expression of FAK (Additional file 2: Fig. 1b), both representing key components of the AKT/PKB survival pathway [28-30], were observed. In addition to these analyses, Western blots were performed on lysates prepared from PDXs at basal conditions to determine the protein levels and activation status of Pan-HER targets EGFR and HER3, as well as the component of their abovementioned signaling pathway, FAK (Fig. 4). Importantly, PDXs for which the response to Pan-HER was most prominent (i.e., subgroup 1) displayed the highest basal levels of pEGFR, pHER3, and FAK (Fig. 4), indicating a potential dependency on these pathways as a survival asset, in the context of which it is

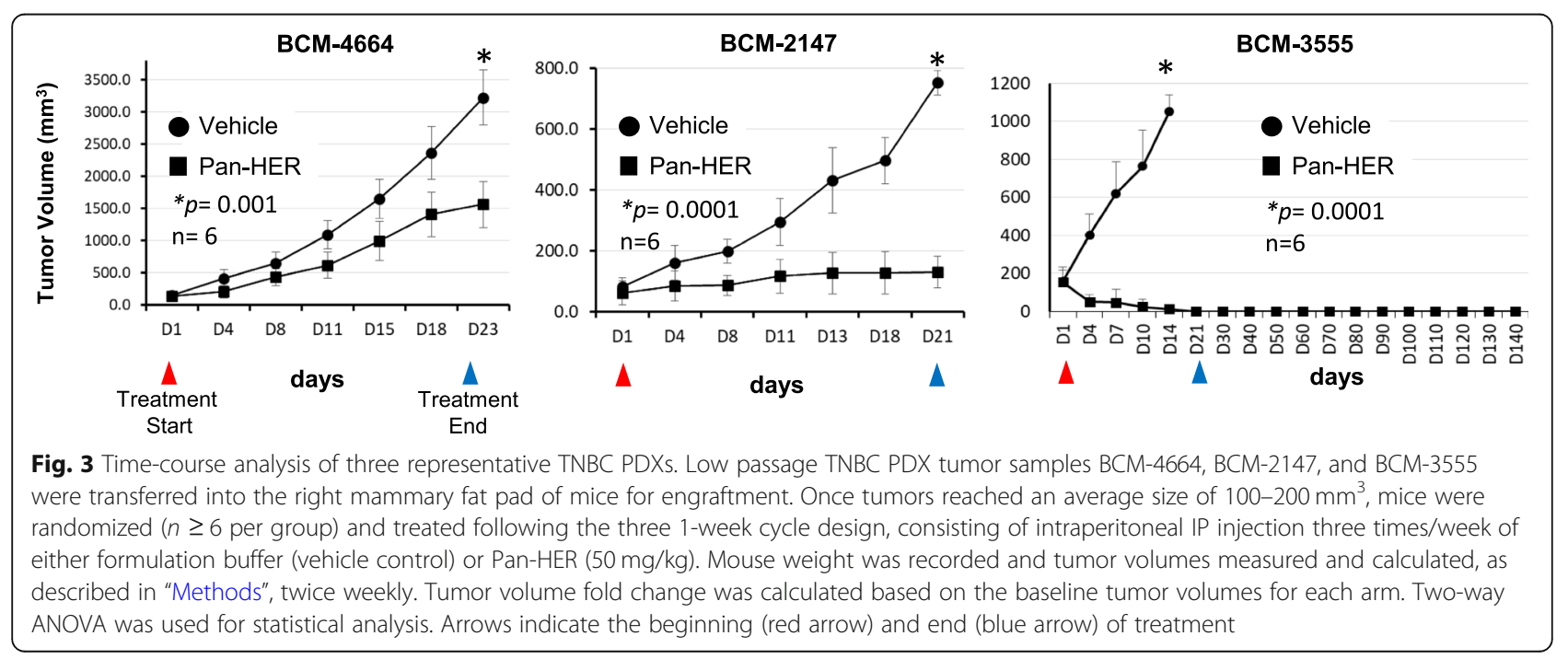




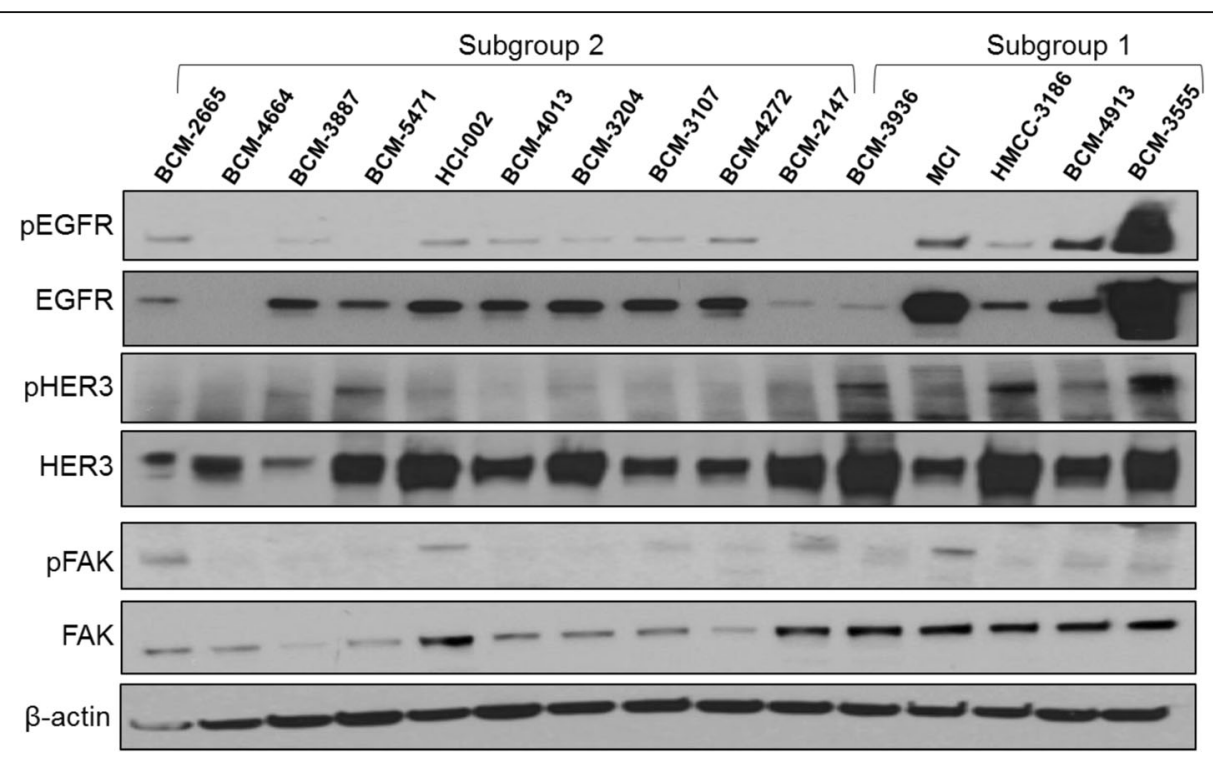

Fig. 4 Analysis of HER family members and associated signaling pathways. Untreated PDX tumor models were analyzed by Western blot to determine the basal, initial levels of expression of HER family members EGFR and HER3, as well as associated signaling pathways including pEGFR, pHER3, PTEN, and FAK. A representative image is shown

plausible to speculate that the simultaneous inhibition of both EGFR and HER3 may account for the increased efficacy of Pan-HER. Noteworthy, the least responsive PDX tumor model, CVM-4663, displayed the lowest, nondetectable level of EGFR expression/activation.

Treatment of TNBC PDXs with Pan-HER dramatically reduces activation of EGFR, HER3, and related signaling pathways

To further determine the mechanisms associated with the Pan-HER activity, analysis of PDX samples treated for 3 cycles with either vehicle control or Pan-HER was performed by both Western blot and immunohistochemistry (Fig. 5 and Additional file 2: Figures S2-S16). Exposure to PanHER resulted in a marked reduction of target pathway activation in both PDX subgroups. Indeed, Pan-HER induced downmodulation of its targets EGFR and HER3 and their corresponding active phosphorylated forms, pEGFR and pHER3 (Fig. 5 and Additional file 2: Figures S2-S16), in agreement with previously reported results in lung and head and neck cancer cells and cetuximab-resistant nonsmall cell lung cancer cells $[4,16,17]$. Consistently, inhibition of EGFR and HER3 negatively affected signaling pathway-associated pFAK, pAKT, and pERK (Fig. 5a). Immunohistochemistry (IHC) analysis provided additional evidence of these differences, showing a significant reduction of both EGFR and HER3 in all Pan-HER-treated samples (Fig. 5b and Additional file 2: Figures S2-S16).

To further evaluate the impact of Pan-HER-meditated inhibition/downregulation of HER family signaling, a pathway-focused, RT-PCR-based analysis of 88 EGFRassociated genes (Additional file Tables: Supplemental Table S4) followed by pathway analysis by IPA (Qiagen) was performed (Fig. 6 and Additional file 2: Figures S17S20). Representative PDX models including BCM-3555 and BCM-4913 (subgroup 1), and BCM-2147 and BCM2665 (subgroup 2) were tested. The RT-PCR analysis was performed using RNA extracted from both vehicle- and Pan-HER-treated tumor tissue collected at the end of the corresponding treatments. Triplicates of each RNA sample, i.e., each PDX model/treatment, were run and analyzed by IPA. As shown in previous figures by IHC and Western blot in all cases, the expression of EGFR was reduced (displayed in green in the corresponding figures). With some differences and/or variations depending the PDX, several genes associated to the EGFR pathway showed also, as expected, significant gene expression downregulation including RAS/cRaf, MEK/ERK, JNK/cJUN/cFOS, and JAK/ STAT3. Furthermore, taking into account that (1) EGF triggers NF- $\kappa B$ activation [37-39] and (2) EGFR/NF- $\kappa B$ pathway crosstalk is known to promote resistance to anticancer therapies [40-43], a similar pathway-focused analysis was performed to establish the effects of Pan-HER on the NF- $\mathrm{kB}$ pathway (Additional file Tables: Supplemental Table S5). As shown in Fig. 6b and Additional file 2: Figures S17-S20, key components of this pathway were affected/ downregulated including forms of IKK, IKB, p65/RelA, and others that would have major impact on the capacity of tumor cells to respond to treatment-related stress. 
a
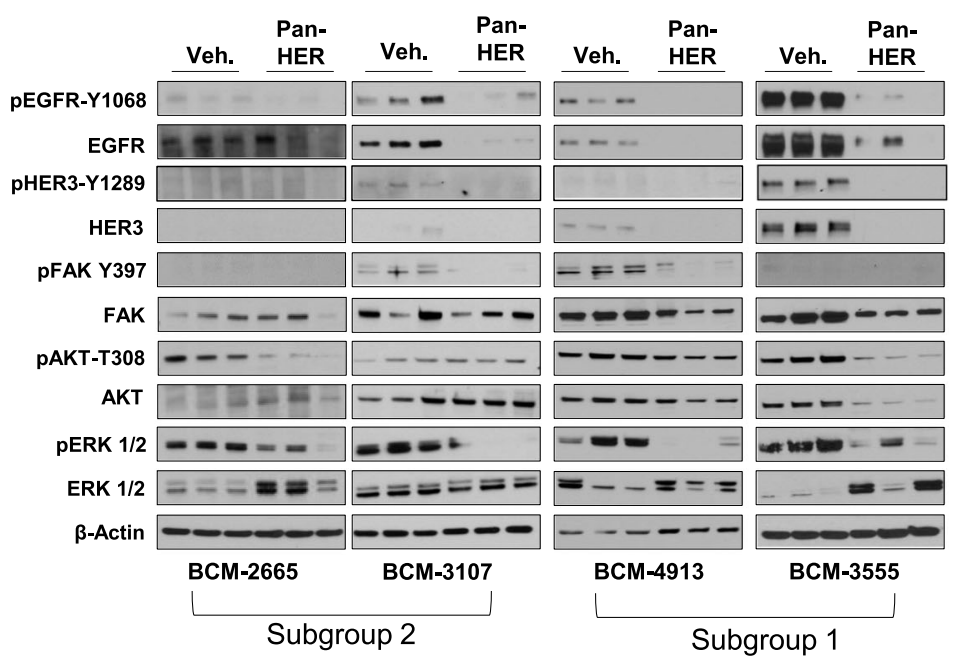

b
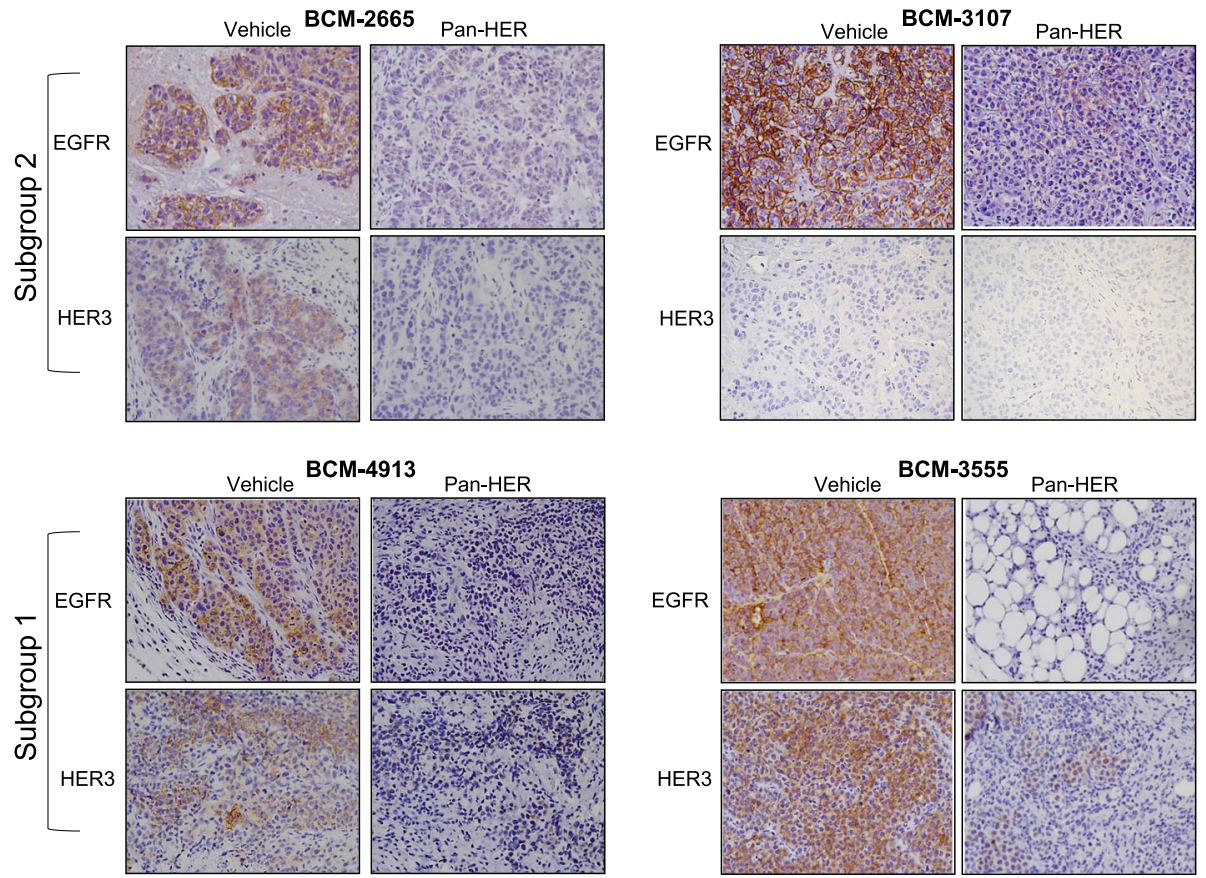

Fig. 5 Analysis of HER family members and associated signaling pathways in representative vehicle- and Pan-HER-treated TNBC PDXs. a Representative PDX tumor models BCM-2665, BCM-3107 (subgroup 2), BCM-4913, and BMC-3555 (subgroup 1) treated with either vehicle control or Pan-HER for 3 cycles were collected at the end of the experiment (day 21 following the initial injection) and analyzed by Western blot (a) or immunohistochemistry (b). Western blot analysis of tumor lysates was used to determine HER family members EGFR and HER3, as well as associated signaling pathways including pEGFR, pHER3, FAK, AKT, and ERK pathways. A representative image is shown. $\beta$-actin levels were used as loading controls. Each Western blot lane corresponds to an individual PDX/mouse of the designated group. b Representative immunohistochemistry analysis of human EGFR and HER3 protein expression was performed in preparations of TNBC tumor samples as described in "Methods"; samples were collected as described above. Counterstain: hematoxylin; here where it says "magnification x 4", it should say "magnification x 20".

Together, these results demonstrate both the effects of Pan-HER administration on specific HER family targets and pro-survival associated signaling pathways as well as the correlation between pathway activation and therapeutic response, indicating their potential use as surrogate biomarkers for patient selection.

\section{Discussion}

The characteristics of TNBC, i.e., absence of ER/PR/HER2 expression, entail that endocrine and HER2-directed therapies are not appropriate for these types of tumors [4446]. Furthermore, TNBC will in most cases develop resistance to anthracyclines and taxanes, both considered to be 

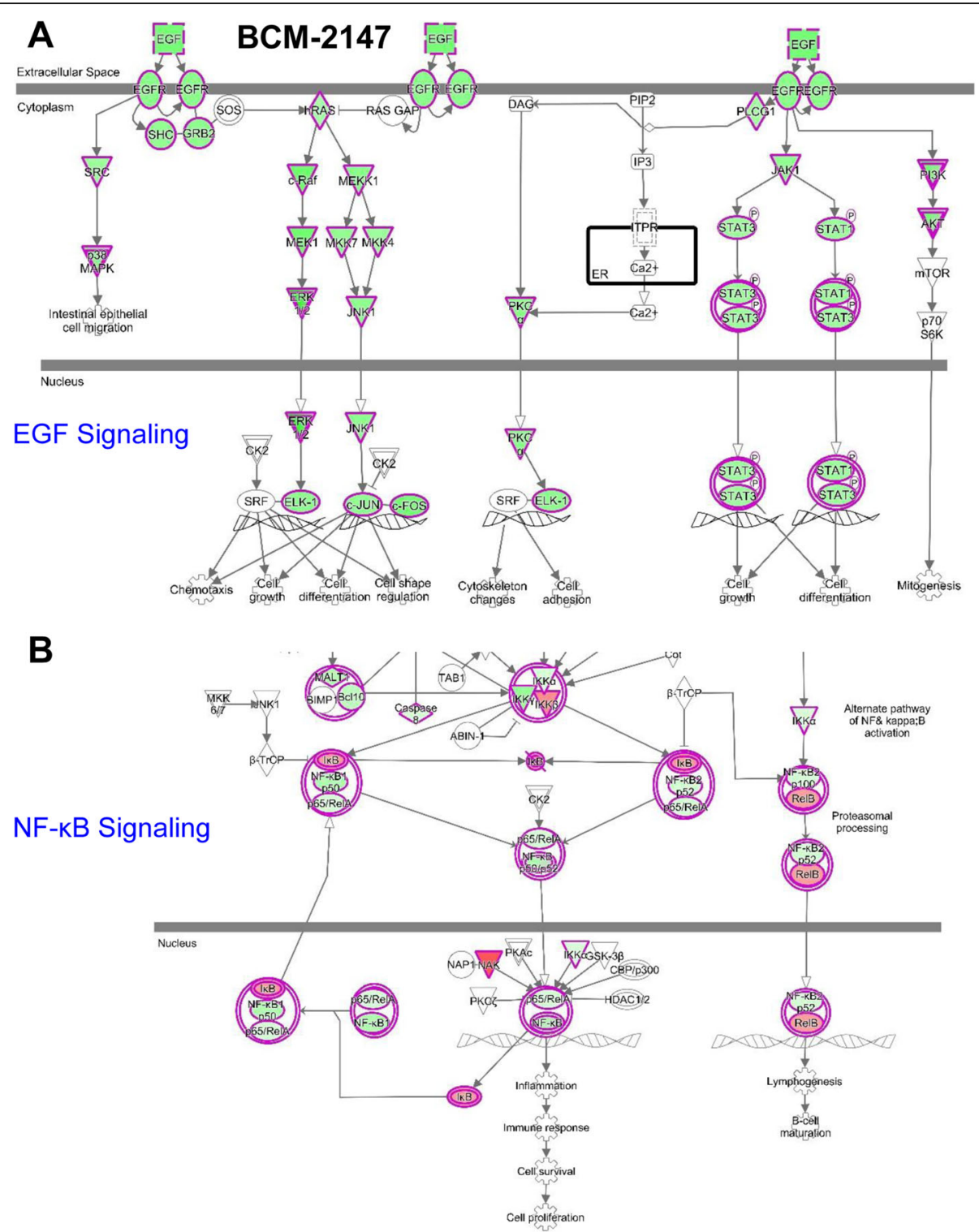

Fig. 6 EGFR (a) and NF-KB (b) pathway-focused RT-PCR gene expression analysis of representative TNBC PDX RNA samples collected before and after Pan-HER treatment. RNA samples corresponding to representative PDX tumor model BCM-2147 (subgroup 2) were extracted from three independent mice (PDX)/group treated with either vehicle control or Pan-HER for 3 cycles at the end of the experiment (day 21 after the initial injection). Triplicate RT-PCR plates were run and relative fold changes of Pan-HER- vs. vehicle control-treated samples for each gene were analyzed by Ingenuity Pathway Analysis (IPA; Qiagen). Genes shown in green represent those significantly downregulated, while those in red upregulated. A twofold change cut-off in gene expression threshold was considered as significantly changed $(P<0.001)$. Further details, as well as a similar analysis performed in three additional PDX models are shown as Supplemental Figures

among the most active compounds against breast cancer, thus limiting the therapeutic options even further [47, 48]. With the discovery several years ago of EGFR being overexpressed in TNBCs, a number of new, targeted therapies were developed, including small-molecule tyrosine kinase inhibitors (TKIs) and mAbs. Several of these are now approved and available, including lapatinib, an oral potent HER2 and EGFR inhibitor [49]; afatinib, also a dual HER2/EGFR inhibitor [50, 51]; neratinib, a pan-HER inhibitor that selectively targets HER2, HER4, and EGFR
$[52,53]$, and the recently introduced erlotinib, an EGFR inhibitor $[54,55]$. Anti-EGFR mAbs include cetuximab and panitumumab, both of which block ligand-induced phosphorylation of EGFR $[56,57]$. However, despite efforts to develop these therapeutic approaches further, these options have not yielded sustained responses and mechanisms of resistance have been encountered after a few months.

In an effort to overcome tumor recurrence and resistance, multiple studies have been addressing the potential use of drug combinations as a way to improve the efficacy 
of treatment. For example, cetuximab has been clinically tested in combination with cisplatin (metastatic TNBC) [58] and carboplatin (stage IV TNBC) [59] and preclinically tested with ixabepilone and other chemotherapeutic agents [60,61]. Additional studies have investigated the concept of targeting simultaneously multiple arms of the same HER family, for example by combining a mAb (cetuximab or panitumumab) with small-molecule TKIs [62-64], and established chemotherapeutic agents [60, 61] or other non-competitive mAbs [13].

In the present study, Pan-HER, a mixture of six antibodies simultaneously targeting EGFR, HER2, and HER3 [4, 15], displayed a very promising activity against TNBC xenografts. TNBCs are characterized by the expression of high levels of EGFR [65-67], and the observation that Pan-HER induced a dramatic downregulation of EGFR and HER3, together with their corresponding signaling pathways, is very relevant. In fact, clinical trials with cetuximab combined with either cisplatin [58] or carboplatin [59] showed only moderate improvements vs. chemotherapy alone in terms of overall response rate and progression-free survival, suggesting that targeting EGFR only, even in combination with other therapies, may not be sufficient. Other studies have expanded in the past on the concept of using the blockade of HER family members $[13,68-70]$. For example, previous studies in TNBC showed the potential therapeutic advantage of blocking both EGFR and HER3, as a way to improve the efficacy of PI3K-Akt inhibitors [14]. Similarly, the combination of non-competitive anti-EGFR mAbs was found to result in a robust degradation of EGFR also in TNBC cell models, demonstrating a reduction on the tumorigenic growth of cells and derived xenografts [13]. In this context, the present results on the efficacy of Pan-HER on TNBC PDXs showed that the mixture of HER family antibodies was very effective in all the PDX models tested, and more pronouncedly in those tumors that displayed highly active HER family expression and signaling, where no recurrence was observed even after several weeks the treatment ended. On the other hand, the least responsive models corresponded to those showing negative or very low expression of either EGFR or HER3, most notably the former. These observations provide not only a plausible explanation for the response of TNBC tumors to Pan-HER but also an indication of the potential use of EGFR and associated signaling expression as biomarkers for selecting patients who may benefit from targeting these proteins, including cases where Pan-HER may be tested in combination with chemotherapies. In this sense, results from a recent trial (I-SPY 2) evaluating the predictive value of the HER/PI3K/AKT signaling activation/ phosphorylation in response to the HER $1 / 2 / 4$ inhibitor neratinib [71], demonstrated that activation of HER family phosphoproteins may be indicative of a response to neratinib.

It is important to note that although not among the most common features observed, relapsed TNBCs have shown some changes in the triple-negative status of the primary tumor type [72]. For example, in a clinical study designed to investigate whether hormonal receptors and HER2 status may be modified throughout tumor progression and therapeutical intervention, it was concluded that these patients experienced changes in the status of hormone receptors and HER2, which could be attributed to adjuvant therapies and may have major impact in survival [72]. Thus, it is plausible to speculate that targeted activation of HER family pathways may help avoid the appearance of resistance. The fact that Pan-HER as a single therapy (inherent combination) was sufficient to achieve complete tumor regression in some tumor models without signs of recurrence makes these findings very promising.

\section{Conclusions}

This preclinical TNBC PDX trial with Pan-HER provides strong justification for further biomarker-guided studies with Pan-HER in TNBC. The finding that tumors were affected rapidly and effectively, with long-lasting results, offers exciting perspectives in the treatment of this aggressive form of breast cancer, for which available treatment options are currently limited.

\section{Supplementary information}

Supplementary information accompanies this paper at https://doi.org/10. 1186/s13058-020-01280-z

Additional file 1. Table 1. Description of the main characteristics of each PDX tumor line are presented including their triple negative (i.e. $\mathrm{ER}^{-}, \mathrm{PR}^{-}$and $\mathrm{HER} 2^{-}$) status, and the positive expression of EGFR and HER3 in all of them, with the exception of BCM-4664, which was HER3Additional information includes, whenever available, TP53 mutations and PAM50 and Pietenpol subtype classification [20, 32-34]. Supplemental Tables 2-3. Sequence analysis of PI3KCA (exons 9 and 20) and EGFR (exons 18, 19, 20 and 21). DNA samples were extracted from all 15 untreated PDX models as described in Additional File Methods. Total DNA was used to perform specific PCR corresponding to PI3KCA (exons 9 and 20) and EGFR (exons 18, 19, 20 and 21). Sequences were analyzed using PI3KCA and EGFR exon sequences from NCBI (EGFR (NM_005228.5) and Multiple Sequence Comparison by Log-Expectation (MUSCLE) program. Supplemental Table 4. Genes included in the pathway-focused RT-PCR analysis corresponding to the human EGFR and NF-kappa B signaling pathways (RealTimePrimers, Elkins Park, PA).

Additional file 2 Figure 1. A, Microarray analysis of RNA gene expression corresponding to PDX samples before treatment. Note the differences in expression of PDXs having shown tumor regression (i.e., BCM-3936, BCM-4913 and MC1; Subgroup 1) vs. the rest of the PDXs (Subgroup 2); PDX BCM-4195 does not express EGFR, HER2, or HER3 and was added to the analysis for comparison only. B, gene expression analysis by Ingenuity Pathway Analysis (Qiagen) comparing BCM-3936, BCM4913 and MC1 (subgroup 1) vs. the remaining PDXs; it shows among the 
top molecules a marked reduction of genes related to the AKT/PKB survival pathway including the PTEN pathway, and increased expression of PTK2 (FAK). Supplemental Figures 2-16. Time course analysis of the therapeutic response corresponding to each of the 15 TNBC PDXs used in the present study. A, graph displaying the time-course analysis of tumor growth; B, Western blot analysis of HER family members and associated signaling events; and $\mathrm{C}, \mathrm{IHC}$ of EGFR and HER3 proteins. Low passage TNBC PDX tumor samples $(2 \mathrm{~mm} \times 2 \mathrm{~mm})$ were transferred into the right mammary fat pad of mice for engraftment. Once tumors reached an average size of $150-200 \mathrm{~mm}^{3}$, mice were randomized ( $n \geq 3$ per group) and treated following the three, one-week cycles design, consisting of 3 times/week IP injection of either formulation buffer (Vehicle control) or Pan-HER (50 mg/kg). Mouse weight was recorded and tumor volumes were measured and calculated as described in Materials \& Methods twice weekly. Tumor volume fold change was calculated based on the baseline tumor volumes for each arm. Two-way ANOVA was used for a statistical analysis. At the end of the 3-cycle treatment, the animals were sacrificed and tumors collected for further Western blot and IHC analyses. Supplemental Figures 17-20. EGFR (A) and NF-KB (B) pathway-focused RT-PCR gene expression analysis of representative TNBC PDXs RNA samples collected before and after Pan-HER treatment. RNA samples corresponding to representative PDX tumor model BCM-2147 and BCM-2665 (Subgroup 2), and BCM-3555 and BCM-4913 (Subgroup 1) were extracted from 3 independent mice( PDX)/group treated with either Vehicle control or PanHER for 3 cycles at the end of the experiment (day 21 after the initial injection). Triplicate RT-PCR plates were run and relative fold changes of Pan-HER- vs. Vehicle control-treated samples for each gene were analyzed by Ingenuity Pathway Analysis (IPA; Qiagen). Genes shown in green represent those significantly down-regulated, while those in red upregulated. A 2 -fold change cut-off in gene expression threshold was considered as significantly changed $(p<0.001)$. Further details, as well as a similar analysis performed in 3 additional PDX models are shown as Supplemental Figures.

Additional file 3. DNA extraction and Sanger sequencing of PIK3CA and EGFR exons

\section{Abbreviations}

HER: Human epidermal growth factor receptor family; TNBC: Triple-negative breast cancer; PDX: Patient-derived xenografts; ERa: Estrogen receptor alpha; PR: Progesterone receptor; HER2: Epidermal growth factor receptor 2; IACUC: Houston Methodist Research Institute Institutional Animal Care and Use Committee; FAK: Focal adhesion kinase; PTEN: Phosphatase and tensin homolog; TCGA: The Cancer Genome Atlas; TKls: Tyrosine kinase inhibitors

\section{Authors' contributions}

DSC, RRR, and JCC conceived of and designed the study in collaboration with JL, IDH, and MK. TR, ACA, WQ, WC, and DSC performed experiments, including animal studies, IHC, RT-PCR, and Western blotting. TR, ACA, DSC, JCC, and RRR performed statistical analysis, interpreted the results, and drafted the manuscript. All authors contributed to the critical revision, editing of the final version of the manuscript, and approval of the final version for publication, and all authors agree to be accountable for the accuracy and integrity of the work.

\section{Funding}

This work was funded by AUP-1010-0025 Chan Soon Shiong Institute of Advanced Health (JCC) and Breast Cancer Research Foundation (BCRF; JCC). Foundation Grants were awarded to JCC for studying novel therapeutics of breast cancer. Additional funding was provided by Symphogen A/S, Ballerup, Denmark.

\section{Availability of data and materials}

All the data supporting the results presented in this article are available upon request at the principal investigator's laboratory.

\section{Ethics approval and consent to participate}

Tissues used to generate the patient-derived xenografts were collected from consenting patients following institutional review board-approved protocols at clinics in the Baylor College of Medicine and Ben Taub General Hospital
(Houston, TX, USA), as previously reported [12], and at Houston Methodist Hospital Cancer Center (protocol Pro00005346).

\section{Consent for publication}

Not applicable, because the present article does not contain any individual person's data in any form.

\section{Competing interests}

Johan Lantto, Ivan D. Horak, and Michael Kragh are co-inventors on a patent owned by Symphogen A/S that describes the Pan-HER composition. The other authors declare that they have no competing interests.

\section{Author details}

${ }^{1}$ Houston Methodist Cancer Center, Houston Methodist Hospital, Houston, TX 77030, USA. ${ }^{2}$ Texas A\&M Health Science Center College of Medicine, Bryan, TX 77807, USA. ${ }^{3}$ Symphogen A/S, Pederstrupvej 93, DK-2750 Ballerup, Denmark.

Received: 26 March 2019 Accepted: 15 April 2020

Published online: 15 May 2020

\section{References}

1. Hammond ME, Hayes DF, Dowsett M, Allred DC, Hagerty KL, Badve S, et al. American Society of Clinical Oncology/College Of American Pathologists guideline recommendations for immunohistochemical testing of estrogen and progesterone receptors in breast cancer. J Clin Oncol. 2010;28(16): 2784-95.

2. Foulkes WD, Smith IE, Reis-Filho JS. Triple-negative breast cancer. N Engl J Med. 2010;363(20):1938-48.

3. Omarini C, Guaitoli G, Pipitone S, Moscetti L, Cortesi L, Cascinu S, Piacentini F. Neoadjuvant treatments in triple-negative breast cancer patients: where we are now and where we are going. Cancer Manag Res. 2018;10:91-103.

4. Jacobsen HJ, Poulsen $\Pi$, Dahlman A, Kjaer I, Koefoed K, Sen JW, et al. PanHER, an antibody mixture simultaneously targeting EGFR, HER2, and HER3, effectively overcomes tumor heterogeneity and plasticity. Clin Cancer Res. 2015;21(18):4110-22.

5. Roskoski R Jr. The ErbB/HER family of protein-tyrosine kinases and cancer. Pharmacol Res. 2014;79:34-74.

6. Balanis N, Carlin CR. Stress-induced EGF receptor signaling through STAT3 and tumor progression in triple-negative breast cancer. Mol Cell Endocrinol. 2017:451:24-30.

7. Brand TM, lida M, Wheeler DL. Molecular mechanisms of resistance to the EGFR monoclonal antibody cetuximab. Cancer Biol Ther. 2011;11(9):777-92.

8. Vlacich G, Coffey RJ. Resistance to EGFR-targeted therapy: a family affair. Cancer Cell. 2011;20(4):423-5.

9. Harris AL, Nicholson S, Sainsbury JR, Farndon J, Wright C. Epidermal growth factor receptors in breast cancer: association with early relapse and death, poor response to hormones and interactions with neu. J Steroid Biochem. 1989;34(1-6):123-31.

10. Citri A, Alroy I, Lavi S, Rubin C, Xu W, Grammatikakis N, et al. Drug-induced ubiquitylation and degradation of ErbB receptor tyrosine kinases: implications for cancer therapy. EMBO J. 2002;21(10):2407-17.

11. Tredan O, Campone M, Jassem J, Vyzula R, Coudert B, Pacilio C, et al. Ixabepilone alone or with cetuximab as first-line treatment for advanced/ metastatic triple-negative breast cancer. Clin Breast Cancer. 2015;15(1):8-15.

12. Tanei T, Choi DS, Rodriguez AA, Liang DH, Dobrolecki L, Ghosh M, Landis MD, Chang JC. Antitumor activity of Cetuximab in combination with Ixabepilone on triple negative breast cancer stem cells. Breast Cancer Res. 2016;18(1):6

13. Ferraro DA, Gaborit N, Maron R, Cohen-Dvashi H, Porat Z, Pareja F, et al. Inhibition of triple-negative breast cancer models by combinations of antibodies to EGFR. Proc Natl Acad Sci U S A. 2013;110(5):1815-20.

14. Tao JJ, Castel P, Radosevic-Robin N, Elkabets M, Auricchio N, Aceto N, et al. Antagonism of EGFR and HER3 enhances the response to inhibitors of the PI3K-Akt pathway in triple-negative breast cancer. Sci Signal. 2014;7(318):ra29.

15. Ellebaek S, Brix S, Grandal M, Lantto J, Horak ID, Kragh M, Poulsen TT. PanHER-an antibody mixture targeting EGFR, HER2 and HER3 abrogates preformed and ligand-induced EGFR homo- and heterodimers. Int J Cancer. 2016;139(9):2095-105. 
16. Francis DM, Huang S, Armstrong EA, Werner LR, Hullett C, Li C, et al. PanHER inhibitor augments radiation response in human lung and head and neck Cancer models. Clin Cancer Res. 2016;22(3):633-43.

17. lida M, Bahrar H, Brand TM, Pearson HE, Coan JP, Orbuch RA, et al. Targeting the HER family with Pan-HER effectively overcomes resistance to cetuximab. Mol Cancer Ther. 2016;15(9):2175-86.

18. Schwarz $\amalg$, Hutchinson KE, Rexer BN, Estrada MV, Gonzalez Ericsson PI, Sanders ME, et al. An ERBB1-3 neutralizing antibody mixture with high activity against drug-resistant HER2+ breast cancers with ERBB ligand overexpression. J Natl Cancer Inst. 2017;109(11)::djx065. https://doi.org/10. 1093/jnci/djx065. https://academic.oup.com/jnci/article/109/11/djx065/3 861234.

19. Koefoed K, Steinaa L, Soderberg JN, Kjaer I, Jacobsen HJ, Meijer PJ, et al. Rational identification of an optimal antibody mixture for targeting the epidermal growth factor receptor. mAbs. 2011;3(6):584-95.

20. Zhang X, Claerhout S, Prat A, Dobrolecki LE, Petrovic I, Lai Q, et al. A renewable tissue resource of phenotypically stable, biologically and ethnically diverse, patient-derived human breast cancer xenograft models. Cancer Res. 2013;73(15):4885-97.

21. Rhodes DR, Kalyana-Sundaram S, Mahavisno V, Varambally R, Yu J, Briggs BB, et al. Oncomine 3.0: genes, pathways, and networks in a collection of 18,000 cancer gene expression profiles. Neoplasia. 2007;9(2):166-80.

22. Dave B, Granados-Principal S, Zhu R, Benz S, Rabizadeh S, Soon-Shiong P, et al. Targeting RPL39 and MLF2 reduces tumor initiation and metastasis in breast cancer by inhibiting nitric oxide synthase signaling. ProcNatlAcadSciUSA. 2014;111(24):8838-43.

23. Gyorffy B, Lanczky A, Eklund AC, Denkert C, Budczies J, Li Q, Szallasi Z. An online survival analysis tool to rapidly assess the effect of 22,277 genes on breast cancer prognosis using microarray data of 1,809 patients. Breast Cancer Res Treat. 2010;123(3):725-31.

24. Gyorffy B, Surowiak P, Budczies J, Lanczky A. Online survival analysis software to assess the prognostic value of biomarkers using transcriptomic data in non-small-cell lung cancer. PLoS One. 2013;8(12):e82241.

25. Granados-Principal S, Liu Y, Guevara ML, Blanco E, Choi DS, Qian W, et al. Inhibition of iNOS as a novel effective targeted therapy against triplenegative breast cancer. Breast Cancer Res. 2015;17:25.

26. Barrett MT, Lenkiewicz E, Malasi S, Basu A, Yearley JH, Annamalai L, et al. The association of genomic lesions and PD-1/PD-L1 expression in resected triple-negative breast cancers. Breast Cancer Res. 2018;20(1):71.

27. Cully M, You H, Levine AJ, Mak TW. Beyond PTEN mutations: the PI3K pathway as an integrator of multiple inputs during tumorigenesis. Nat Rev Cancer. 2006;6(3):184-92.

28. Daragmeh J, Barriah W, Saad B, Zaid H. Analysis of PI3K pathway components in human cancers. Oncol Lett. 2016;11(4):2913-8.

29. Park JH, Cho YY, Yoon SW, Park B. Suppression of MMP-9 and FAK expression by pomolic acid via blocking of NF-kappaB/ERK/mTOR signaling pathways in growth factor-stimulated human breast cancer cells. Int J Oncol. 2016;49(3):1230-40.

30. Mertins P, Mani DR, Ruggles KV, Gillette MA, Clauser KR, Wang P, et al. Proteogenomics connects somatic mutations to signalling in breast cancer. Nature. 2016;534(7605):55-62.

31. Huang PH, Xu AM, White FM, Oncogenic EGFR. signaling networks in glioma. Sci Signal. 2009;2(87):re6.

32. Landis MD, Lehmann BD, Pietenpol JA, Chang JC. Patient-derived breast tumor xenografts facilitating personalized cancer therapy. Breast Cancer Res. 2013;15(1):201.

33. Lehmann BD, Pietenpol JA. Identification and use of biomarkers in treatment strategies for triple-negative breast cancer subtypes. J Pathol. 2014;232(2):142-50.

34. Nielsen TO, Parker JS, Leung S, Voduc D, Ebbert M, Vickery T, et al. A comparison of PAM50 intrinsic subtyping with immunohistochemistry and clinical prognostic factors in tamoxifen-treated estrogen receptor-positive breast cancer. Clin Cancer Res. 2010;16(21):5222-32.

35. Peifer M, Fernandez-Cuesta L, Sos ML, George J, Seidel D, Kasper LH, et al. Integrative genome analyses identify key somatic driver mutations of smallcell lung cancer. Nat Genet. 2012;44(10):1104-10.

36. George J, Lim JS, Jang SJ, Cun Y, Ozretic L, Kong G, et al. Comprehensive genomic profiles of small cell lung cancer. Nature. 2015;524(7563):47-53.

37. Biswas DK, Cruz AP, Gansberger E, Pardee AB. Epidermal growth factorinduced nuclear factor kappa B activation: a major pathway of cell-cycle progression in estrogen-receptor negative breast cancer cells. Proc Natl Acad Sci U S A. 2000;97(15):8542-7.
38. Sun L, Carpenter G. Epidermal growth factor activation of NF-kappaB is mediated through IkappaBalpha degradation and intracellular free calcium. Oncogene. 1998;16(16):2095-102.

39. Shostak K, Chariot A. EGFR and NF-kappaB: partners in cancer. Trends Mol Med. 2015;21(6):385-93.

40. Bentires-Alj M, Barbu V, Fillet M, Chariot A, Relic B, Jacobs N, Gielen J, Merville MP, Bours V. NF-kappaB transcription factor induces drug resistance through MDR1 expression in cancer cells. Oncogene. 2003;22(1):90-7.

41. Bivona TG, Hieronymus H, Parker J, Chang K, Taron M, Rosell R, et al. FAS and NF-kappaB signalling modulate dependence of lung cancers on mutant EGFR. Nature. 2011;471(7339):523-6.

42. Dermawan JK, Gurova K, Pink J, Dowlati A, De S, Narla G, Sharma N, Stark GR. Quinacrine overcomes resistance to erlotinib by inhibiting FACT, NFkappaB, and cell-cycle progression in non-small cell lung cancer. Mol Cancer Ther. 2014;13(9):2203-14.

43. Tanaka K, Babic I, Nathanson D, Akhavan D, Guo D, Gini B, et al. Oncogenic EGFR signaling activates an mTORC2-NF-kappaB pathway that promotes chemotherapy resistance. Cancer Discov. 2011;1(6):524-38.

44. Jamdade VS, Sethi N, Mundhe NA, Kumar P, Lahkar M, Sinha N. Therapeutic targets of triple-negative breast cancer: a review. Br J Pharmacol. 2015; 172(17):4228-37.

45. Marme F. Schneeweiss A. Targeted therapies in triple-negative breast cancer. Breast Care (Basel). 2015;10(3):159-66.

46. Yadav BS, Sharma SC, Chanana P, Jhamb S. Systemic treatment strategies for triple-negative breast cancer. World J Clin Oncol. 2014;5(2):125-33.

47. Andre F, Zielinski CC. Optimal strategies for the treatment of metastatic triple-negative breast cancer with currently approved agents. Ann Oncol. 2012;23(Suppl 6):vi46-51.

48. Arnedos M, Bihan C, Delaloge S, Andre F. Triple-negative breast cancer: are we making headway at least? Ther Adv Med Oncol. 2012;4(4):195-210.

49. Rusnak DW, Lackey K, Affleck K, Wood ER, Alligood KJ, Rhodes N, et al. The effects of the novel, reversible epidermal growth factor receptor/ErbB-2 tyrosine kinase inhibitor, GW2016, on the growth of human normal and tumor-derived cell lines in vitro and in vivo. Mol Cancer Ther. 2001;1(2):8594.

50. Li D, Ambrogio L, Shimamura T, Kubo S, Takahashi M, Chirieac LR, et al. BIBW2992, an irreversible EGFR/HER2 inhibitor highly effective in preclinical lung cancer models. Oncogene. 2008;27(34):4702-11.

51. Schuler M, Awada A, Harter P, Canon JL, Possinger K, Schmidt M, et al. A phase II trial to assess efficacy and safety of afatinib in extensively pretreated patients with HER2-negative metastatic breast cancer. Breast Cancer Res Treat. 2012;134(3):1149-59.

52. Feldinger K, Kong A. Profile of neratinib and its potential in the treatment of breast cancer. Breast Cancer (Dove Med Press). 2015;7:147-62.

53. Mullooly M, Conklin D, McGowan PM, O'Brien NA, O'Donovan N, Slamon DJ, Crown J, Finn RS, Duffy MJ. Neratinib to inhibit the growth of triplenegative breast cancer cells. J Clin Oncol. 2015;33(15_suppl):1099.

54. Maurer M, Kalinsky K, Forman J, Jayasena R, Crew K, Hershman D Abstract OT1-4-03: Phase I study of erlotinib and metformin in triple negative breast cancer. Cancer Res. 2013;73(24 Supplement):OT1-4-03-OT1-4-

55. Nagaria TS, Shi C, Leduc C, Hoskin V, Sikdar S, Sangrar W, Greer PA. Combined targeting of Raf and Mek synergistically inhibits tumorigenesis in triple negative breast cancer model systems. Oncotarget. 2017;8(46):80804-19.

56. Prewett MC, Hooper AT, Bassi R, Ellis LM, Waksal HW, Hicklin DJ. Enhanced antitumor activity of anti-epidermal growth factor receptor monoclonal antibody IMC-C225 in combination with irinotecan (CPT-11) against human colorectal tumor xenografts. Clin Cancer Res. 2002;8(5):994-1003.

57. Costa R, Shah AN, Santa-Maria CA, Cruz MR, Mahalingam D, Carneiro BA, et al. Targeting epidermal growth factor receptor in triple negative breast cancer: new discoveries and practical insights for drug development. Cancer Treat Rev. 2017;53:111-9.

58. Baselga J, Gomez P, Greil R, Braga S, Climent MA, Wardley AM, et al. Randomized phase II study of the anti-epidermal growth factor receptor monoclonal antibody cetuximab with cisplatin versus cisplatin alone in patients with metastatic triplenegative breast cancer. J Clin Oncol. 2013;31(20):2586-92.

59. Carey LA, Rugo HS, Marcom PK, Mayer EL, Esteva FJ, Ma CX, et al. TBCRC 001: randomized phase II study of cetuximab in combination with carboplatin in stage IV triple-negative breast cancer. J Clin Oncol. 2012; 30(21):2615-23.

60. Tanei T, Morimoto K, Shimazu K, Kim SJ, Tanji Y, Taguchi T, Tamaki Y, Noguchi S. Association of breast cancer stem cells identified by aldehyde 
dehydrogenase 1 expression with resistance to sequential paclitaxel and epirubicin-based chemotherapy for breast cancers. ClinCancer Res. 2009; 15(12):4234-41.

61. Nakai K, Hung MC, Yamaguchi H. A perspective on anti-EGFR therapies targeting triple-negative breast cancer. Am J Cancer Res. 2016;6(8):1609-23.

62. Huang S, Armstrong EA, Benavente S, Chinnaiyan P, Harari PM. Dual-agent molecular targeting of the epidermal growth factor receptor (EGFR): combining anti-EGFR antibody with tyrosine kinase inhibitor. Cancer Res. 2004;64(15):5355-62.

63. Matar P, Rojo F, Cassia R, Moreno-Bueno G, Di Cosimo S, Tabernero J, et al. Combined epidermal growth factor receptor targeting with the tyrosine kinase inhibitor gefitinib (ZD1839) and the monoclonal antibody cetuximab (IMC-C225): superiority over single-agent receptor targeting. Clin Cancer Res. 2004;10(19):6487-501.

64. El Guerrab A, Bamdad M, Bignon YJ, Penault-Llorca F, Aubel C. Anti-EGFR monoclonal antibodies enhance sensitivity to DNA-damaging agents in BRCA1-mutated and PTEN-wild-type triple-negative breast cancer cells. Mol Carcinog. 2017;56(5):1383-94.

65. Lerma E, Peiro G, Ramon T, Fernandez S, Martinez D, Pons C, et al. Immunohistochemical heterogeneity of breast carcinomas negative for estrogen receptors, progesterone receptors and Her2/neu (basal-like breast carcinomas). Mod Pathol. 2007:20(11):1200-7.

66. Pintens S, Neven P, Drijkoningen M, Van Belle V, Moerman P, Christiaens MR, Smeets A, Wildiers H, Vanden Bempt I. Triple negative breast cancer: a study from the point of view of basal CK5/6 and HER-1. J Clin Pathol. 2009;62(7): 624-8.

67. Rakha EA, El-Sayed ME, Green AR, Lee AH, Robertson JF, Ellis IO. Prognostic markers in triple-negative breast cancer. Cancer. 2007;109(1):25-32.

68. Spangler JB, Neil JR, Abramovitch S, Yarden Y, White FM, Lauffenburger DA, Wittrup KD. Combination antibody treatment down-regulates epidermal growth factor receptor by inhibiting endosomal recycling. Proc Natl Acad Sci U S A. 2010;107(30):13252-7.

69. Ben-Kasus T, Schechter B, Lavi S, Yarden Y, Sela M. Persistent elimination of ErbB-2/HER2-overexpressing tumors using combinations of monoclonal antibodies: relevance of receptor endocytosis. Proc Natl Acad Sci U S A. 2009;106(9):3294-9.

70. Friedman LM, Rinon A, Schechter B, Lyass L, Lavi S, Bacus SS, Sela M, Yarden Y. Synergistic down-regulation of receptor tyrosine kinases by combinations of mAbs: implications for cancer immunotherapy. Proc Natl Acad Sci U S A. 2005;102(6):1915-20

71. Wulfkuhle JD, Yau C, Wolf DM, Vis DJ, Gallagher RI, Brown-Swigart L, et al. Evaluation of the HER/PI3K/AKT family signaling network as a predictive biomarker of pathologic complete response for patients with breast cancer treated with Neratinib in the I-SPY 2 TRIAL. JCO Precision Oncol. 2018:2:1-20.

72. Lindstrom LS, Karlsson E, Wilking UM, Johansson U, Hartman J, Lidbrink EK, Hatschek T, Skoog L, Bergh J. Clinically used breast cancer markers such as estrogen receptor, progesterone receptor, and human epidermal growth factor receptor 2 are unstable throughout tumor progression. J Clin Oncol. 2012;30(21):2601-8.

\section{Publisher's Note}

Springer Nature remains neutral with regard to jurisdictional claims in published maps and institutional affiliations.

Ready to submit your research? Choose BMC and benefit from:

- fast, convenient online submission

- thorough peer review by experienced researchers in your field

- rapid publication on acceptance

- support for research data, including large and complex data types

- gold Open Access which fosters wider collaboration and increased citations

- maximum visibility for your research: over $100 \mathrm{M}$ website views per year

At BMC, research is always in progress.

Learn more biomedcentral.com/submissions 